\title{
Editorial
}

\section{"Smoke gets in your eyes"}

\section{Liebe Kolleginnen und Kollegen!}

Das Jahr 2008 wird für die Ophthalmologie ein Jahr mehrerer Aktivitäten werden. Wir haben uns vorgenommen, die Ausbildung der jungen Kollegen in den nächsten Jahren so zu strukturieren, dass sie einem internationalen Vergleich standhalten kann. Hierfür werden wir die Richtlinien des European Boards of Ophthalmology verwenden und auch auf die Erfahrung der American Academy of Ophthalmology zurückgreifen. Die österreichische Ophthalmologie wird sich dem weltweiten Projekt „Vision 2020“ anschließen, das auch einen nationalen Part inkludiert. Es gilt, die Zahl der Blinden bzw. Sehbehinderten auch in unserem Land zu reduzieren und entsprechende Aktionen zu initiieren, die Risikofaktoren aufzeigen.

Einer dieser Risikofaktoren, der ganz oben auf der Liste der beeinflussbaren Ursachen für Altersbedingte Makuladegeneration steht - die ja die größte Krankengruppe von Sehbehinderung im Alter darstellt -, ist das Rauchen. Große Studien in Australien und Neuseeland haben gezeigt, dass Rauchen die Inzidenz einer altersbedingten Makuladegeneration verdoppelt. Genetische Studien konnten zeigen, dass bei entsprechender genetischer Disposition das Risiko eines Menschen, der zusätzlich noch raucht, auf das 8-fache ,ansteigt“.

Nach einer Umfrage der britischen Ophthalmologen haben junge Menschen im Alter zwischen 25 und 40 Jahren auf die Frage „Fürchten Sie beim Rauchen eher die Komplikation des Lungenkrebses oder die Kompli- kation der Erblindung“ zu 81\% geantwortet, dass sie sich vor der Erblindung weitaus mehr fürchten würden.

Die Gruppe, die das Rauchen wegen der Angst vor der Erblindung aufgeben würde, war daher wesentlich höher als jene Gruppe, die sich vorm Sterben fürchten.

Die Österreichische Ophthalmologische Gesellschaft wurde aufgefordert, sich an dieser Kampagne zu beteiligen, was wir in einem Beschluss des Vorstandes im November 2007 auch getan haben. Neben der Veröffentlichung verschiedener Daten in den Medien bezüglich des erhöhten Risikos der Erblindung oder Sehbehinderung beim Rauchen soll auch die Forderung gestellt werden, einen entsprechenden Vermerk auf den Zigarettenpackungen beizufügen. Im nächsten Jahr werden wir Ihnen noch laufend über diese Aktion berichten.

Das vorliegende Heft beinhaltet besonders zwei interessante Artikel, einen zum Thema der Kurzsichtigkeit und einen zum Thema des diabetischen Makulaödems. Für beide Erkrankungen haben wir trotz ausführlicher Forschung leider noch keine endgültige Lösung gefunden.

Ich wünsche Ihnen für die kommenden Feiertage ein friedliches Weihnachtsfest und alles Gute für das Jahr 2008.

Mit kollegialer Hochachtung e. h. Univ.-Prof. Dr. Susanne Binder 